\title{
Sigma-Pi 神经网络中的逼近问题*
}

\author{
李纯明陈天平 \\ (复旦大学数学研究所, 上海 200433)
}

\section{关键词逼近论 Sigma-Pi 神经网络 稠密性}

设函数 $\pi: \mathbb{R}^{n} \rightarrow$ 呑定义如下:

$$
\pi:\left(x_{1}, \cdots, x_{n}\right) \mapsto \prod_{k=1}^{n} x_{k} .
$$

近来, 用一个一元连续函数 $g$ 与函数 $\pi$ 的复合 $g \circ \pi$ 来逼近多元连续问题. 由于其在人工神经 网络中的重要应用,而受到广泛的关注 ${ }^{[1 \sim 4]}$.

事实上, 所谓 Sigma-Pi 神经网络的能力, 从数学上讲, 就是形如

$$
\sum_{i=1}^{N} c_{i} g \cdot \pi\left(\lambda_{i} x-t^{(i)}\right)
$$

的函数全体在 $C(K)$ 中稠密性问题, 其中 $K$ 为涩 $^{H}$ 中的一个紧集, $C_{i}, \lambda_{i} \in \mathbb{R}, t^{(i)} \in \mathbb{R}^{h}, x \in K$. 如 果一个函数 $g$ 使得形如 (1) 式的函数全体在 $C(K)$ 中稠密, 则 $g$ 可以作为 Sigma-Pi 神经网络 的激发函数.

一个重要的问题是: 什么样的一元函数可以作为 Sigma-Pi 神经网络的激发函数?

最近, 在文献[4] 中, Lenze 证明了

定理 $\mathrm{A}$ 设 $\sigma$ 是一个连续的 Sigmoidal 函数, 即满足

的连续函数, 则形如

$$
\lim _{u \rightarrow-\infty} \sigma(u)=0, \lim _{u \rightarrow \infty} \sigma(u)=1
$$

$$
\sum_{i=1}^{N} C_{i} \sigma \cdot \pi\left(\lambda_{i} x-t^{(i)}\right)
$$

的函数全体在 $C(K)$ 中稠密.

在本文中, 我们证明了

定理 设 $g:$ 冒 $\rightarrow$ 冒是一个连续函数, 且满足

(1) 存在 $\delta>0$, 使函数 $g$ 在 $(-\delta, \delta)$ 中无穷次可微;

(2) 存在一列正整数 $p_{1}<p_{2}<\cdots<p_{k}<\cdots$, 使得

$$
\sum_{k=1}^{\infty} \frac{1}{p_{k}}=\infty \text { 且 } g^{\left(p_{k}\right)}(0) \neq 0, k=1,2, \cdots,
$$

1995-7-13 收稿, 1995-11-13 收修改稿

*国家教委博士点基金及国家自然科学基金资助项目 
则形如 (1) 式的函数全体在 $C(K)$ 中稠密.

注 在实际应用中, 许多常用的函数均满足定理中的条件 (1) 及 (2), 如 $\mathrm{e}^{x}, \mathrm{e}^{-x^{2}}, \cos ^{x}$, $\sin x,\left(1+x^{2}\right)^{-1},\left(1+\mathrm{e}^{-x}\right)^{-1}, \cdots$. 因此, 上述定理表明, 这些函数均可以作为 Sigma-Pi 神经网络 的激发函数.

为了证明定理, 引进一列函数 $g_{k}(x)=|x| x^{k}$, 其中 $k$ 为非负整数, 且记 $\alpha_{k}=(k, \cdots, k)$ 为 $n$ 重 指标. $D^{\alpha_{k}}$ 为通常的偏微分算子.

定理的证明基于如下几个引理:

引理 1 设 $k$ 为非负整数, 则对任意 $f \in C_{c}^{\infty}\left(\mathbb{R}^{n}\right)$, 成立着

$$
f(x)=(-1)^{n k}[2(k+1) !]^{-n} \int_{\mathbb{R}^{n}} g_{k} \circ \pi(x-t)\left(D^{\alpha_{k}} f\right)(t) \mathrm{d} t .
$$

应用引理 1 , 可以证明

引理 2 设 $k$ 为非负整数,则形如

$$
\sum_{i=1}^{N} c_{i} g_{k} \circ \pi\left(\lambda_{i} x-t^{(i)}\right)
$$

的函数全体在 $C(K)$ 中稠密.

应用 Müntz 定量, 可以证得

引理 3 设偶整数列 $0=p_{1}<p_{2}<\cdots<p_{k}<\cdots$ 满足 $\sum_{k=1}^{\infty} \frac{1}{p_{k}}=\infty$, 则下述函数

$$
c_{0}+\sum_{i=1}^{N} c_{i} x^{p_{i}}
$$

全体在 $C[-r, r]$ 中稠密, 其中 $r$ 为任意正实数.

引理 4 设 $r>0, g$ :㘣 $\rightarrow$ 回为一连续函数, 且满足定理中的条件 (1) 及 (2), 则存在非负整 数 $p$, 使得函数 $g_{p}(x)$ 可以被形如

$$
\sum_{i=1}^{N} c_{i} g\left(\lambda_{i} x\right), c_{i}, \quad \lambda_{i} \in R, i=1, \cdots, N
$$

的函数在 $[-r, r]$ 中一致逼近.

结合上述 4 个引理, 通过一些技巧及细致的计算, 可以证明定理.

本文定理表明了, 相当广泛的一类函数可以作为 Sigma-Pi 神经网络的激发函数. 然而,一 个尚未解决的理论问题是找出作为 Sigma-Pi 神经网络激发函数的充要条件.

\section{参考文献}

1 Chen Tinanping, Chen Hong. Approximations of continuous functionals by neural networks with applications to dynamic system. IEEE Transactions on Neural Networks, 1993, 4(6): $96 \sim 918$

2 Chen Tianping, Chen Hong, Liu Ruey-wen. Approximation capability in $C\left(\overline{\mathbb{R}}^{n}\right)$ by multilayer feedforward :networks and related problems. IEEE Transactions on Neural Networks, 1995, 6(1): 25 30

3 陈天平. 神经网络及其在系统识别中的逼近问题. 中国科学, A 辑, 1994, 24(1): 1 7

4 Lenze B. Note on a density question for neural networks. Numerical Functional Analysis And Optimization, 1994, 15 (7 \& 8): $909 \sim 913$

5 切尼 E W. 逼近论导引. 上海 : 上海科学技术出版社, 1981 